\title{
Spectroscopic Investigation of the Mixture of Ascorbic Acid and Sodium Benzoate
}

\author{
Femi Francis Oloye \\ Department of Chemical Sciences, Adekunle Ajasin University, Akungba Akoko, Nigeria
}

Email address:

femi.oloye@aaua.edu.ng

\section{To cite this article:}

Femi Francis Oloye. Spectroscopic Investigation of the Mixture of Ascorbic Acid and Sodium Benzoate. Science Journal of Chemistry. Vol. 7, No. 3, 2019, pp. 62-66. doi: 10.11648/j.sjc.20190703.12

Received: July 31, 2019; Accepted: August 20, 2019; Published: September 12, 2019

\begin{abstract}
UV spectroscopy was employed to understand the possible interaction between ascorbic acid (AA) and sodium benzoate (SB). The absorbance of each of the preservatives was taken singly (285 and $291 \mathrm{~nm}$ for SB and AA, respectively) and the spectrum of a common drink that contained the two preservatives was recorded. Then the interaction of AA with SB was monitored by varying the concentration of $\mathrm{AA}$ in $\mathrm{SB}$, reaction temperature and exposure to sunlight. The peaks of AA and SB disappeared and a new peak emerged at higher wavelength upon addition of AA to SB, suggesting a redshift and incorporation of AA in SB. Sunlight and temperature ( $\leq$ body temperature) did not cause AA and SB to react or changes in the wavelength of maximum absorbance. Addition of AA into a typical fizzy drink did not result in new peak suggesting AA did not create any new products by its addition to this product, which already contained AA and SB at the recommended level. However, the addition of a higher amount of AA into the fizzy drinks cause a noticeable red shift from 287.5 to $295 \mathrm{~nm}$. Thus caution should be taken when taking AA with SB containing products.
\end{abstract}

Keywords: Spectroscopy, Ascorbic Acid, Sodium Benzoate, Preservatives, Spectrum, Concentration, Red Shift

\section{Introduction}

Several studies in chemistry focus on the interaction between two substances [1-4] however little is known about the interaction of food additives. Food additives are substances added to preserve flavor, or to enhance food taste and appearance, during any of the following stages; production, packaging, transportation and/or storage [5]. Examples of the commonly used additives are monosodium glutamate (MSG), white sugar, benzoic acid (and its salts, such as potassium and sodium benzoate), ascorbic acid (vitamin C), aluminium silicate, butylated hydroxyl toluene (BHT), etc. [6]. Sodium benzoate is commonly used as an antimicrobial in pickles, sauces, fruit juices, pharmaceuticals, and therapeutic regimens [7]. It is also used as preservative agents in edible coatings [7]. The acceptable daily limit intake of benzoic acid and its salts range between $0-5 \mathrm{mg} / \mathrm{kg}$ body weights [8]. Ascorbic acid is an important antioxidant with multiple cellular functions.

Spectroscopy is a common method to monitor chemical reaction that occurred when two or more chemical interacts together. No classical chemical study can give as much information as the spectroscopic studies, in a very short time without destroying the compound [9]. UV radiations are absorbed by different compounds at different wavelengths because of electronic transition that occurs within the $\pi$ conjugated molecules. If there is a change in how UV radiation was absorbed between two chemical substances then any of the two common types of interaction are responsible. These interactions are; increase/decrease in absorbance with an appearance of a new band, which indicates the formation of a new compound or intermediate; and increase/decrease in extinction coefficient with a red/blue shift, which indicates incorporation of one additive into another [2].

Science has achieved a lot of success in dye-surfactant interaction because such interaction has helped in understanding the chemical equilibria, mechanisms, and kinetics of surfactant-sensitized colour and /or fluorescence reactions [2]. Surfactant is generally amphiphiles possessing distinct regions of hydrophobic and hydrophilic characteristics due to their chemical structure of the hydrophilic moiety bound to the hydrophobic portion [10]. Hence, this surfactants can interact with organic compounds 
(hydrophobic end) as well as polar substance (water related, with hydrophilic end). Sodium benzoate is an example of anionic surfactant, which has both hydrophobic and hydrophilic characteristics. These surfactant molecules form aggregates at higher amphiphile concentrations $\left(>10^{-4} \mathrm{M}\right)$, which are called micelles, and these micelles are efficient solubilizers of different organic molecules in water, including ascorbic acid, dyes, etc. [10-13]. Surfactant generally interacts with organic molecules through any of the following interaction; electrostatic, hydrophobic and charge transfer

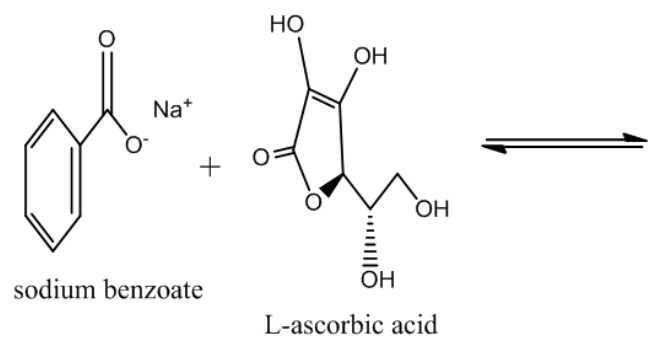

L-ascorbic acid interaction [8]. Figure 1 shows the possible reaction between sodium benzoate and ascorbic acid to produce benzoic acid and sodium ascorbate. The reaction may proceed forward to give benzene and carbon (IV) oxide if the benzoic acid dissociates. This study will involve the use of UV to monitor the type of shift that will occur as a result of the reaction of sodium benzoate with ascorbic acid in water. This investigation can give useful information as regards the thermodynamics and kinetics of preservatives combination in the food industry.

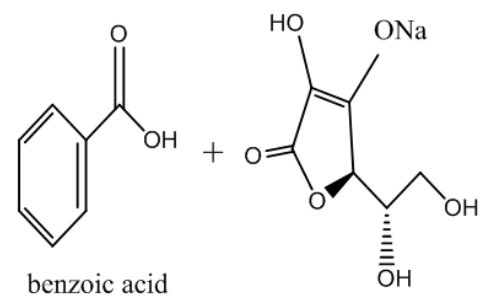

Sodium L-ascorbate

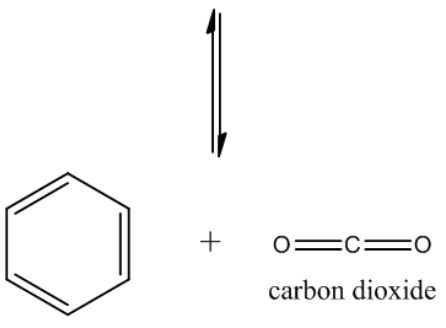

benzene

Figure 1. Propose reaction pathway for the reaction of sodium benzoate with ascorbic acid.

\section{Material and Methods}

All chemicals were of analytical reagent grade. Sodium benzoate (SB) and ascorbic acid (AA) were purchased from Sigma Aldrich, UK. Solutions were prepared using distilled water. A matched pair of quartz cuvette $(1.00 \mathrm{~cm}$ path length) with UV-Visible Spectrophotometer (UV-1902PC) was used for all spectroscopic measurements. The absorption spectra of sodium benzoate solution and ascorbic acid solution were taken separately and their maximum absorbance $\left(\lambda_{\max }\right)$ was recorded as 285 and $291 \mathrm{~nm}$, respectively. The reproducibility of $\lambda_{\max }$ of the spectral was greater than $0.1 \mathrm{~nm}$. All measurements were done in triplicate.

In this study, the interaction of SB with AA, which could result in the formation of intermediate, was determined based on the changes in the $\lambda_{\max }$ of SB and AA. The change in the $\lambda_{\max }$ absorbance of SB and AA was monitored by varying the concentration of AA $(0.011 \mathrm{M}, 0.023 \mathrm{M}$, and $0.034 \mathrm{M})$ by using distilled water as the reference at room temperature. The variation of $\lambda_{\max }$ absorbance in the range of $190-1100 \mathrm{~nm}$ was recorded.

A solution of $0.014 \mathrm{M} \mathrm{SB}$ with a solution of $0.011 \mathrm{M} \mathrm{AA}$ was used to understand the influence of temperature on the interactions of $\mathrm{SB}$ and $\mathrm{AA}$. The temperature were varied between the room temperature and body temperature (298, $300,303,306,309$ and $312 \mathrm{~K})$. The effects of sun lights were recorded by keeping the same concentration in the lab and another one in the sun, then taken the maximum absorbance at every $30 \mathrm{mins}$. Furthermore, to understand the role of increase concentration of ascorbic acid on the properties of food already preserved with ascorbic acid and sodium benzoate, a known food (carbonated drinks) with both AA and SB was used as a model example. Varied amounts of AA were added to a known concentration of the fizzy drink and stirred together for 30 mins and then the spectra of the samples were taken.

\section{Results and Discussion}

The correlations between absorbance and concentration for AA $\left(\mathrm{R}^{2}=0.9997\right)$ and SB $\left(\mathrm{R}^{2}=0.9696\right)$ (in figures A1 and A2, respectively) were linear, this indicates the acceptability of Beer-Lambert law at the studied concentrations level [14]. Thus the absorbance can easily be converted to concentration. The $\lambda_{\max }$ of SB and AA are 285 and $291 \mathrm{~nm}$ in distilled water (spectra not shown). Upon reaction of varied concentration of AA with constant concentration of $(0.014 \mathrm{M})$ $\mathrm{SB}$, the $\lambda_{\max }$ increased from both 285 and $291 \mathrm{~nm}$ to 298 , 299.5 and $300 \mathrm{~nm}$ (table 1) as the concentration of AA increased from $0.011,0.023,0.034 \mathrm{M}$ (table 1). The red shift observed with the addition of varied concentration of AA with SB suggested incorporation of AA to SB. A blue shift was not observed at any situations, which could be an indication that there is no molecular complex formation 
between the $\mathrm{AA}$ and $\mathrm{SB}$, which might have been resulted from electromagnetic interaction [2]. SB being an example of a surfactant has unique structural feature, with which it is able to establish interactions with both the hydrophilic as well as hydrophobic (organic, such as AA) molecules [1]. Hence, there is possibility of interaction between SB and AA. Organic compounds that are poorly soluble in water could be solubilized by both ionic and cationic surfactant when they aggregates by incorporating them in the micellar phase [1]. These micelles are conveniently exploited to act as catalysts for many reactions due to their large surface area; they are capable to alter the reaction pathways, rates and equilibria [1], so many different possibilities are possible when working with high concentration of SB $\left(>10^{-4} \mathrm{M}\right)$. Since there is no evidence of formation of an additional peak (s) (in figure 2), then the form of interaction that occurs between $\mathrm{AA}$ and $\mathrm{SB}$ at the study concentration might just be the incorporation of AA in SB or SB in AA [15].

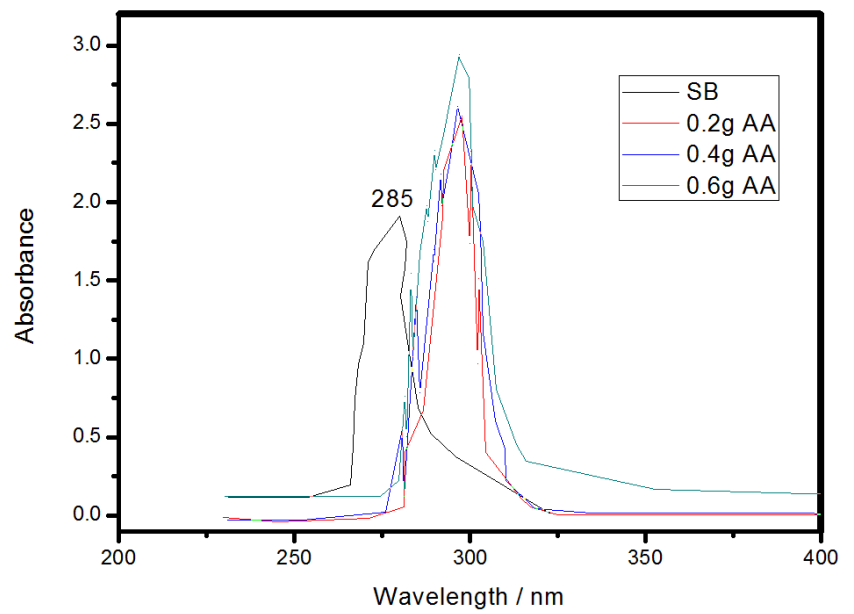

Figure 2. Spectra obtained from varied concentration of AA in 0.0139 moldm ${ }^{-3} \mathrm{SB}$ in water.

Table 1. A representative yield and maximum absorbance of varied concentration of $A A$ in 0.0139 moldm $^{-3} S B$ in water $(n=3)$.

\begin{tabular}{lll}
\hline $\mathbf{A A}\left(\mathbf{m o l} / \mathbf{d m}^{\mathbf{3}}\right)$ & $\left(\mathbf{C}_{\mathbf{S B}}+\mathbf{C}_{\mathbf{A A}}\right)^{-1}$ & $\boldsymbol{\lambda}_{\max (\mathbf{n m})}$ \\
\hline 0.011 & 40.180 & $298 \pm 0.001$ \\
0.023 & 27.109 & $299.5 \pm 0.001$ \\
0.034 & 20.882 & $300 \pm 0.002$ \\
\hline
\end{tabular}

Table 2. Effect of temperature on $S B(0.014 M)$ with $A A(0.011 M)$ in water $(n=3)$.

\begin{tabular}{lll}
\hline Temperature $(\mathbf{K})$ & $\boldsymbol{\lambda}_{\max } \pm$ SD $(\mathbf{n m})$ & Absorbance \pm SD $(\mathbf{n m})$ \\
\hline 298 & $298 \pm 0.0010$ & $2.8504 \pm 0.0194$ \\
300 & $298 \pm 0.0008$ & $2.8302 \pm 0.0200$ \\
303 & $298.5 \pm 0.0009$ & $2.8501 \pm 0.0195$ \\
306 & $299 \pm 0.0011$ & $2.8102 \pm 0.0220$ \\
309 & $299.5 \pm 0.0010$ & $2.8583 \pm 0.0190$ \\
312 & $301 \pm 0.0012$ & $2.9242 \pm 0.0010$ \\
\hline
\end{tabular}

Table 3. Wavelength and absorbance of Fanta (100mL) with varied concentration of ascorbic acid $(n=3)$.

\begin{tabular}{lll}
\hline Ascorbic Acid $(\mathbf{g})$ & $\boldsymbol{\lambda}_{\max (\mathrm{nm})}$ & $\boldsymbol{\Lambda}(\mathbf{n m})$ \\
\hline 0.0 & $287.5(0.5449 \pm 0.011)^{*}$ & $481.5(0.6372 \pm 0.01)^{*}$ \\
0.1 & $288.5(1.6600 \pm 0.012)^{*}$ & $481.5(0.7019 \pm 0.01)^{*}$ \\
\hline
\end{tabular}

\begin{tabular}{lll}
\hline Ascorbic Acid $(\mathbf{g})$ & $\boldsymbol{\lambda}_{\max (\mathrm{nm})}$ & $\boldsymbol{\Lambda}(\mathbf{n m})$ \\
\hline 0.2 & $290.0(1.7753 \pm 0.013)^{*}$ & $481.5(0.5756 \pm 0.02)^{*}$ \\
0.3 & $290.5(1.8770 \pm 0.011)^{*}$ & $481.5(0.6262 \pm 0.01)^{*}$ \\
0.4 & $291.0(1.9430 \pm 0.012)^{*}$ & $481.5(0.5867 \pm 0.02)^{*}$ \\
0.5 & $294.0(2.0800 \pm 0.011)^{*}$ & $481.5(0.6464 \pm 0.02)^{*}$ \\
\hline
\end{tabular}

*Indicate the corresponding absorbance value \pm SD.

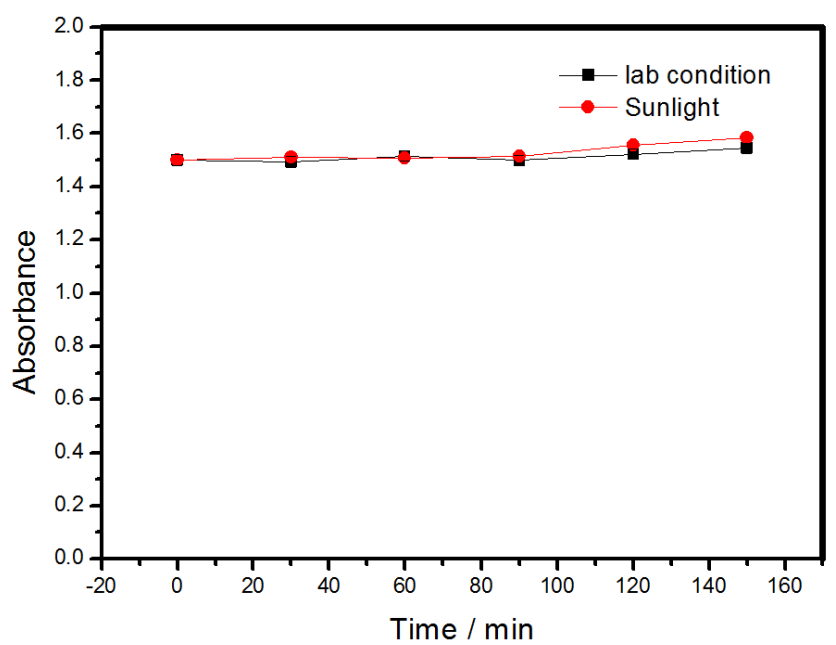

Figure 3. Sodium benzoate $(0.2 \mathrm{~g} / \mathrm{ml})$ with ascorbic acid $(0.4 \mathrm{~g} / \mathrm{ml})$ under sunlight irradiation and normal lab condition $(n=3)$.

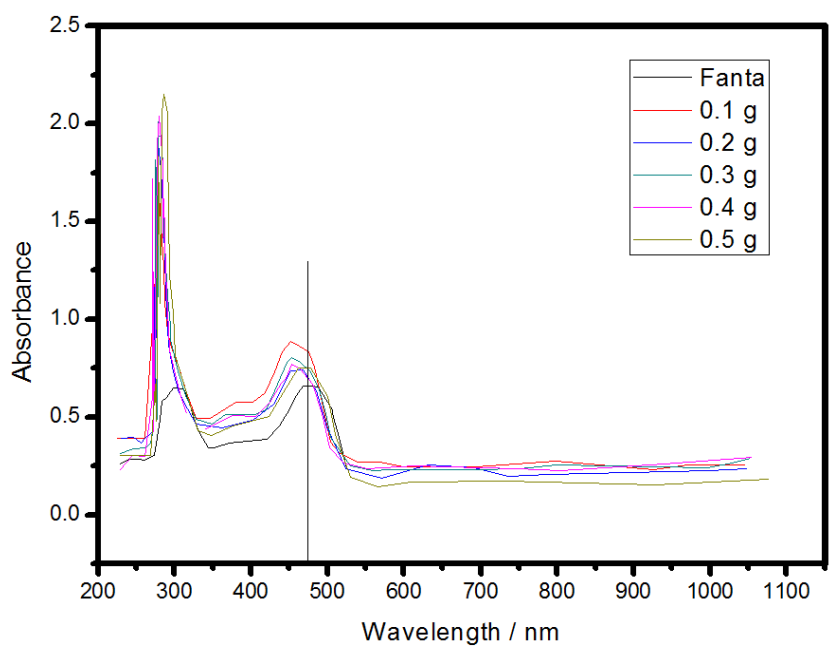

Figure 4. UV spectra of Fanta $(100 \mathrm{~mL})$ with varied concentration of ascorbic acid.

The effect of normal room temperature as well as average body temperature was tested on the mixture of SB and AA. It is evident from table 2 that no major change was observable as the temperature was changed from $298 \mathrm{~K}$ (room temperature), 300, 303, 306, 309 (approximate body temperature) and $312 \mathrm{~K}$. This suggests that $\mathrm{SB}$ and $\mathrm{AA}$ at low concentrations will not interact under room temperature or within body temperature. Another attempt was made to understand the effect of sunlight, and it was observed that sunlight did not alter the maximum absorbance wavelength or intensity. The absorbance remained the constant for over $2 \mathrm{~h}$ (figure 3 ). Thus suggesting that low concentration of SB and AA will not react because of sunlight illumination.

A representative of common drinks (fanta) that contain 
both SB and AA within acceptable limit is shown in figure 4. Two major peaks and a minor peak were evident at 287,378 (minor) and $481 \mathrm{~nm}$. The first peak at 287 drift slightly with the increase in AA concentration and the absorbance also increased with the concentration of AA (table 3). However, the peak at $481 \mathrm{~nm}$ did not change position and intensity, suggesting the main component of Fanta. The rate of change in maximum absorbance wavelength in Fanta upon addition of AA is similar to what was obtain with $\mathrm{SB}$ and AA (table 1). This is an indication that incorporation of $\mathrm{AA}$ in $\mathrm{SB}$ or $\mathrm{SB}$ in AA occurred. As the weight of AA increased to $0.5 \mathrm{~g}$ a clear shift in the absorption towards red shift occurred, suggesting the possibility of interaction. Hence the concentration of AA in SB should be kept at a minimal concentration to avoid possible interactions.

\section{Conclusion}

Ascorbic acid (AA) and sodium benzoate (SB) did not interact to form new product (s) at minimal concentrations but incorporate into each other, this was evident by the red shift as the concentration of AA in SB increased. Sunlight did not lead to the interaction of AA and SB also temperature below body temperature $(310 \mathrm{~K})$ did not cause a significant change in maximum absorbance wavelength. Addition of AA into a typical fizzy drink did not result in new peak suggesting AA did not create any new products by its addition to this product which already contained $\mathrm{AA}$ and $\mathrm{SB}$ at the recommended level. However, the addition of a high amount of AA into the fizzy drinks cause a noticeable red shift from 287.5 to $295 \mathrm{~nm}$. Thus caution should be taken when taking AA with SB containing products.

\section{Appendix}

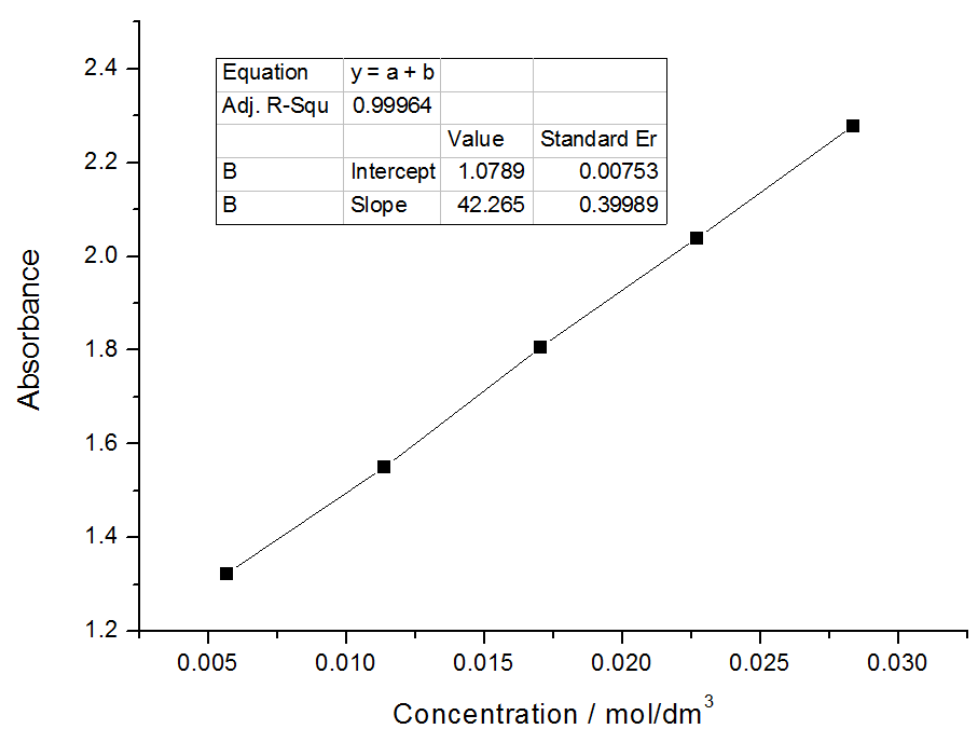

Figure A1. Plot of absorbance against ascorbic acid concentration.

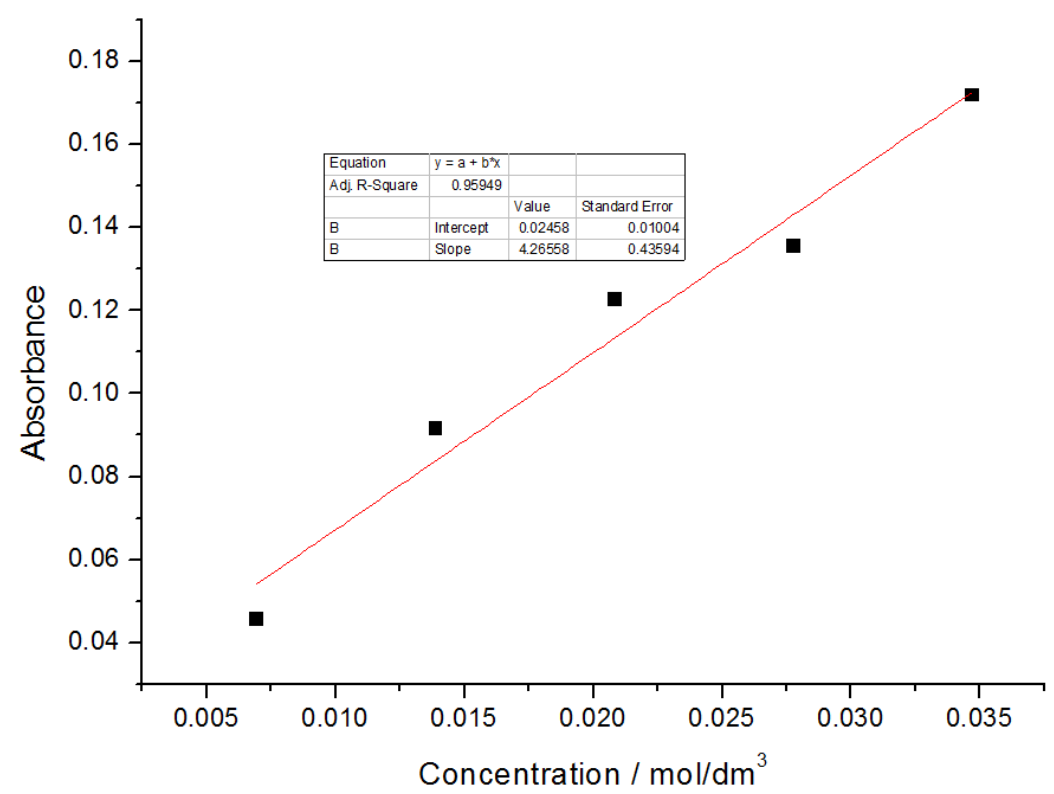

Figure A2. Plot of absorbance against sodium benzoate concentration. 


\section{Acknowledgements}

The Tertiary Education Trust Fund, Nigeria is appreciated for funding the research.

\section{References}

[1] Ali A, Uzair S, Malik NA, Ali M. Study of interaction between cationic surfactants and cresol red dye by electrical conductivity and spectroscopy methods. Journal of Molecular Liquids, 2014; 196: 395-403.

[2] EFSA Scientific Opinion on the re-evaluation of benzoic acid (E 210), sodium benzoate (E 211), potassium benzoate (E 212) and calcium benzoate (E 213) as food additives. European Food Safety Authority Journal, 2016; 14 (3): 44334559. 33 .

[3] Gokturk S, Tuncay M., Dye-surfactant interaction in the premicellar region. J. Surf. Deterg. 2003: 6 (4), 325-30.

[4] Erdainç N, Göktürk S, Tunçay M. Interaction of epirubicin $\mathrm{HCl}$ with surfactants: Effect of $\mathrm{NaCl}$ and glucose. Journal Pharmaceutical Science 2004; 1, 93 (6): 1566-1576.

[5] Kunkel, EM, Barbara, HD. Gale Nutrition and Well-being A to Z. The Gale Group Inc., Macmilln Refernce USA, New York, 2004.

[6] Abdulmumeen HA, Risikat AN, Sururah AR. Food: its preservatives additives applications. International Journal of Chemical and Biochemical Sciences 2012; 1: 36-47.
[7] CICAD Benzoic acid and sodium benzoate. Concise International Chemical Assessment Document, 2005, 26: 146.

[8] Tewari KS, Vishnoi NK. A textbook of Organic Chemistry. VIKAS, 2011.

[9] Gokturk S, Tuncay M. Spectral studies of safranin-O in different surfactant solutions. Spectrochimica Acta Part A 2003; 59: 1857-1866.

[10] Pande S, Ghosh SJ, Nath S, Praharaj S, Jana S, Panigrahi S, Basu S, Pal T. Reduction of methylene blue by thiocyanate: Kinetic and thermodynamic aspects. Journal of Colloid and Interface Science 2006; 299: 421-427.

[11] Jean YC, Ache HJ. Positronium reactions in micellar systems. Journal of America Chemical Society 1977; 99 (23): 75047509 .

[12] Pal T, Jana NR. Polarity dependent positional shift of probe in a micellar environment. Langmuir 1996; 26, 12 (13): 3114-21.

[13] Zhang G, and Ma Y. Spectroscopic studies on the interaction of sodium benzoate, a food preservative, with calf thymus DNA. Food Chemistry 2013; 1, 141 (1): 41-7.

[14] Göktürk S, Yeşim Talman R, Erdinç N, Tunçay M. Solution behaviour of rivanol in micellar environments. Spectroscopic Letters. 2006; 39 (4): 357-372.

[15] Yu J, Liu JY, Xiong WM, Zhang XY, Zheng Y. Binding interaction of sodium benzoate food additive with bovine serum albumin: multi-spectroscopy and molecular docking studies. BMC Chemistry. 2019; 13 (1): 95-103. 